\title{
Fractional Gradient Elasticity from Spatial Dispersion Law
}

\author{
Vasily E. Tarasov \\ Skobeltsyn Institute of Nuclear Physics, Lomonosov Moscow State University, Moscow 119991, Russia \\ Correspondence should be addressed to Vasily E. Tarasov; tarasov@theory.sinp.msu.ru \\ Received 4 February 2014; Accepted 10 March 2014; Published 3 April 2014 \\ Academic Editors: V. Kochereshko, A. A. Kordyuk, A. Krimmel, V. Stephanovich, and S. Wang
}

Copyright (C) 2014 Vasily E. Tarasov. This is an open access article distributed under the Creative Commons Attribution License, which permits unrestricted use, distribution, and reproduction in any medium, provided the original work is properly cited.

\begin{abstract}
Nonlocal elasticity models in continuum mechanics can be treated with two different approaches: the gradient elasticity models (weak nonlocality) and the integral nonlocal models (strong nonlocality). This paper focuses on the fractional generalization of gradient elasticity that allows us to describe a weak nonlocality of power-law type. We suggest a lattice model with spatial dispersion of power-law type as a microscopic model of fractional gradient elastic continuum. We demonstrate how the continuum limit transforms the equations for lattice with this spatial dispersion into the continuum equations with fractional Laplacians in Riesz's form. A weak nonlocality of power-law type in the nonlocal elasticity theory is derived from the fractional weak spatial dispersion in the lattice model. The continuum equations with derivatives of noninteger orders, which are obtained from the lattice model, can be considered as a fractional generalization of the gradient elasticity. These equations of fractional elasticity are solved for some special cases: subgradient elasticity and supergradient elasticity.
\end{abstract}

\section{Introduction}

The theory of derivatives and integrals of noninteger orders [1-3] allows us to investigate the behavior of materials and media that are characterized by nonlocality of power-law type. Fractional calculus has a wide application in mechanics and physics (e.g., see [4-14]). Nonlocal elasticity theories in continuum mechanics can be treated with two different approaches [15]: the gradient elasticity theory (weak nonlocality) and the integral nonlocal theory (strong nonlocality). The fractional calculus allows us to formulate a fractional generalization of nonlocal elasticity models in two forms: the fractional gradient elasticity models (weak power-law nonlocality) and the fractional integral nonlocal models (strong power-law nonlocality). The idea to include some fractional integral term in the equations of the elasticity has been proposed by Lazopoulos in [16]. Fractional models of integral nonlocal elasticity are considered in different papers; see, for example, [16-22]. The microscopic models of fractional integral elasticity are also described. For this reason, the fractional integral elasticity models are not discussed here.

This paper focuses on the fractional generalization of gradient elasticity which describes a weak nonlocality of power type. We suggest a lattice model with spatial dispersion of power-law type as a microscopic model of fractional gradient elastic continuum. Complex lattice dynamics has been the subject of continuing interest in the theory of elasticity. As it was shown in [23, 24] (see also [25-27]), the equations with fractional derivatives can be directly connected to lattice models with long-range interactions. In this paper, we consider models of lattices with spatial dispersion and its continuous limits. We define a map of lattice models into continuum models. A connection between the dynamics of lattice system of particles with longrange interactions and the fractional continuum equations is proved by using the transform operation [23, 24]. We make the transformation to the continuous limit and derive the fractional equation, which describes the dynamics of the nonlocal elastic materials. We show how the continuous limit for the lattice with fractional weak spatial dispersion gives the corresponding continuum equation of the fractional gradient elasticity. The continuum equations of fractional elasticity are solved for some special cases: subgradient elasticity and supergradient elasticity.

\section{Lattice Equations}

The lattice is characterized by space periodicity. In an unbounded lattice, we can define three noncoplanar vectors 
$\mathbf{a}_{1}, \mathbf{a}_{1}, \mathbf{a}_{1}$, such that displacement of the lattice by the length of any of these vectors brings it back to itself. The vectors $\mathbf{a}_{i}, i=1,2,3$, are the shortest vectors by which a lattice can be displaced and be brought back into itself. As a result, all spatial lattice points can be defined by the vector $\mathbf{n}=$ $\left(n_{1}, n_{2}, n_{3}\right)$, where $n_{i}$ are integer. If we choose the coordinate origin at one of the sites, then the position vector of an arbitrary lattice site with $\mathbf{n}=\left(n_{1}, n_{2}, n_{3}\right)$ is written:

$$
\mathbf{r}(\mathbf{n})=\sum_{i=1}^{3} n_{i} \mathbf{a}_{i}
$$

In a lattice, the sites are numbered in the same way as the particles, so that the vector $\mathbf{n}$ is at the same time "number vector" of a corresponding particle.

We assume that the equilibrium positions of particles coincide with the lattice sites $\mathbf{r}(\mathbf{n})$. A lattice site coordinate $\mathbf{r}(\mathbf{n})$ differs from the coordinate of the corresponding particle, when particles are displaced relative to their equilibrium positions. To define the coordinates of a particle, it is necessary to indicate its displacement with respect to its equilibrium positions. We denote the displacement of a particle with vector $\mathbf{n}$ from its equilibrium position by the vector field $\mathbf{u}(\mathbf{n}, t)$.

The equation of motion of lattice particle is

$$
M \frac{\partial^{2} u^{k}(\mathbf{n}, t)}{\partial t^{2}}=-\sum_{\mathbf{m}} K_{k l}(\mathbf{n}, \mathbf{m}) u^{l}(\mathbf{m}, t)+F_{k}(\mathbf{n}, t),
$$

where $M$ is the mass of particle and $F_{k}(\mathbf{n}, t)$ are components of the external on-site force. The italics $k, l$ are the coordinate indices. We assume the summation over doubly repeated coordinate indices from 1 to 3 . The coefficients $K_{k l}(\mathbf{n}, \mathbf{m})$ describe the interparticle interaction in the lattice. For simplicity, we assume that all particles have the same mass $M$.

It is easy see one important property of the coefficients $K_{k l}(\mathbf{n}, \mathbf{m})$. Assume the lattice to be displaced as a whole: $u^{k}(\mathbf{n}, t)=u^{k}=$ constant. Then the internal lattice state cannot be changed in case of absence of external forces. As a result, (2) gives

$$
\sum_{\mathbf{m}} K_{k l}(\mathbf{n}, \mathbf{m})=\sum_{\mathbf{m}} K_{k l}(\mathbf{m}, \mathbf{n})=0 .
$$

These conditions should be satisfied for any particle in the lattice, that is, for any vector $\mathbf{n}$. Equation (3) follows from the conservation of total momentum in the lattice.

For an unbounded homogeneous lattice, due to its homogeneity the matrix $K_{k l}(\mathbf{n}, \mathbf{m})$ has the form

$$
K_{k l}(\mathbf{n}, \mathbf{m})=K_{k l}(\mathbf{n}-\mathbf{m}),
$$

where elements of $K_{k l}(\mathbf{n}-\mathbf{m})$ of (2) are satisfied by condition

$$
\sum_{\mathbf{m}} K_{k l}(\mathbf{n}-\mathbf{m})=\sum_{\mathbf{n}} K_{k l}(\mathbf{n}-\mathbf{m})=0 .
$$

In a simple lattice, each particle is an inversion center, and we have

$$
K_{k l}(\mathbf{n}-\mathbf{m})=K_{k l}(\mathbf{m}-\mathbf{n}) .
$$

Using condition (5), we can represent (2) in the form

$$
\begin{aligned}
M \frac{\partial^{2} u^{k}(\mathbf{n}, t)}{\partial t^{2}}= & -\sum_{\mathbf{m}} K_{k l}(\mathbf{n}, \mathbf{m})\left(u^{l}(\mathbf{n}, t)-u^{l}(\mathbf{m}, t)\right) \\
& +F_{k}(\mathbf{n}, t) .
\end{aligned}
$$

This equation of motion has the invariance with respect to its displacement of lattice as a whole in case of absence of external forces even if condition (5) is not satisfied. It should be noted that the noninvariant terms lead to the divergences in the continuous limit [13].

Equation of motion (7) is equation for three-dimensional displacement vectors. In this paper, we will use the simplest model to describe the lattice, where all particles are displaced in one direction; we assume that the displacement of particle from its equilibrium position is determined by a scalar rather than a vector. This model allows us to describe the main properties of the lattice using simple equations.

The equations of motion for one-dimensional lattice system of interacting particles have the form

$$
M \frac{\partial^{2} u_{n}(t)}{\partial t^{2}}=g \sum_{\substack{m=-\infty \\ m \neq n}}^{+\infty} K_{\alpha}(n, m)\left(u_{n}(t)-u_{m}(t)\right)+F(n),
$$

where we use the summation condition over repeated indexes. Here $u_{n}(t)=u(n, t)$ are displacements from the equilibrium, $g$ is the coupling constant for interparticle interactions in the lattice, and the term $F(n)$ characterizes an interaction of the particles with the external on-site force.

\section{Transform Operations for Lattice Equations}

Let us define the operation that transforms the lattice equations for $u_{n}(t)$ into the continuum equation for a scalar field $u(x, t)$. In order to obtain continuum equation from the lattice equations, we assume that $u_{n}(t)$ are Fourier coefficients of some function $\widehat{u}(k, t)$. We define the field $\widehat{u}(k, t)$ on $\left[-k_{0} / 2, k_{0} / 2\right]$ by the equation

$$
\begin{gathered}
\widehat{u}(k, t)=\sum_{n=-\infty}^{+\infty} u_{n}(t) e^{-i k x_{n}}=\mathscr{F}_{\Delta}\left\{u_{n}(t)\right\}, \\
u_{n}(t)=\frac{1}{k_{0}} \int_{-k_{0} / 2}^{+k_{0} / 2} d k \widehat{u}(k, t) e^{i k x_{n}}=\mathscr{F}_{\Delta}^{-1}\{\widehat{u}(k, t)\},
\end{gathered}
$$

where $x_{n}=n d$ and $d=2 \pi / k_{0}$ is the interparticle distance. For simplicity, we assume that all particles have the same interparticle distance $d$. Equations (9) can be used to obtain the Fourier transform in the limit $d \rightarrow 0\left(k_{0} \rightarrow \infty\right)$. Then change the sum to an integral, and (9) become

$$
\begin{gathered}
\tilde{u}(k, t)=\int_{-\infty}^{+\infty} d x e^{-i k x} u(x, t)=\mathscr{F}\{u(x, t)\}, \\
u(x, t)=\frac{1}{2 \pi} \int_{-\infty}^{+\infty} d k e^{i k x} \tilde{u}(k, t)=\mathscr{F}^{-1}\{\tilde{u}(k, t)\} .
\end{gathered}
$$


We replace the discrete function

$$
u_{n}(t)=\frac{2 \pi}{k_{0}} u\left(x_{n}, t\right)
$$

by continuous field $u(x, t)$ considering $x_{n}=n d=2 \pi n / k_{0} \rightarrow$ $x$. We assume that $\widetilde{u}(k, t)=\mathscr{L} \widehat{u}(k, t)$, where $\mathscr{L}$ denotes the passage to the limit $d \rightarrow 0\left(k_{0} \rightarrow \infty\right)$. Here $\tilde{u}(k, t)$ is a Fourier transform of the field $u(x, t)$ and $\widehat{u}(k, t)$ is a Fourier series transform of $u_{n}(t)$, where we use $u_{n}(t)=$ $\left(2 \pi / k_{0}\right) u(n d, t)$. The function $\widetilde{u}(k, t)$ can be derived from $\widehat{u}(k, t)$ in the limit $d \rightarrow 0$.

As a result, we define the map from a lattice model into a continuum model by the transform operation $\widehat{T}$, which is the combination $[23,24] \widehat{T}=\mathscr{F}^{-1} \mathscr{L} \mathscr{F}_{\Delta}$ of the following operations:

(1) the Fourier series transform:

$$
\mathscr{F}_{\Delta}: u_{n}(t) \longrightarrow \mathscr{F}_{\Delta}\left\{u_{n}(t)\right\}=\widehat{u}(k, t),
$$

(2) the passage to the limit $d \rightarrow 0$ :

$$
\mathscr{L}: \widehat{u}(k, t) \longrightarrow \mathscr{L}\{\widehat{u}(k, t)\}=\widetilde{u}(k, t),
$$

(3) the inverse Fourier transform:

$$
\mathscr{F}^{-1}: \tilde{u}(k, t) \longrightarrow \mathscr{F}^{-1}\{\tilde{u}(k, t)\}=u(x, t) .
$$

The similar transformations can be performed for differential equations to map the lattice equation into an equation for the elastic continuum. Therefore, the operation $\widehat{T}=$ $\mathscr{F}^{-1} \mathscr{L} \mathscr{F}_{\Delta}$ allows us to realize transformation of lattice models of interacting particles into continuum models [23, 24].

Let us consider the Fourier series transform of the interaction term.

Proposition 1. Let $K_{\alpha}(n, m)$ be such that conditions

$$
\begin{gathered}
K_{\alpha}(n, m)=K_{\alpha}(n-m)=K_{\alpha}(m-n), \\
\sum_{n=1}^{\infty}\left|K_{\alpha}(n)\right|^{2}<\infty
\end{gathered}
$$

hold. Then the Fourier series transform $\mathscr{F}_{\Delta}$ maps the term

$$
\sum_{\substack{m=-\infty \\ m \neq n}}^{+\infty} K_{\alpha}(n, m)\left(u_{n}(t)-u_{m}(t)\right)
$$

where $u_{n}=u_{n}(t)$ is a position of the $n$th particle, into the term

$$
\begin{gathered}
\mathscr{F}_{\Delta}\left(\sum_{\substack{m=-\infty \\
m \neq n}}^{+\infty} K_{\alpha}(n, m)\left(u_{n}(t)-u_{m}(t)\right)\right) \\
=\left(\widehat{K}_{\alpha}(0)-\widehat{K}_{\alpha}(k d)\right) \widehat{u}(k, t),
\end{gathered}
$$

where

$$
\widehat{K}_{\alpha}(k d)=\mathscr{F}_{\Delta}\left\{K_{\alpha}(n)\right\}, \quad \widehat{u}(k, t)=\mathscr{F}_{\Delta}\left\{u_{n}(t)\right\} .
$$

Proof. To derive the Fourier series transform of the interaction term (16), we multiply (16) by $\exp (-i k n d)$ and sum over $n$ from $-\infty$ to $+\infty$. Then

$$
\begin{gathered}
\sum_{n=-\infty}^{+\infty} \sum_{\substack{m=-\infty \\
m \neq n}}^{+\infty} e^{-i k n d} K_{\alpha}(n-m)\left(u_{n}-u_{m}\right) \\
=\sum_{n=-\infty}^{+\infty} \sum_{\substack{m=-\infty \\
m \neq n}}^{+\infty} e^{-i k n d} K_{\alpha}(n-m) u_{n} \\
\quad-\sum_{n=-\infty}^{+\infty} \sum_{\substack{m=-\infty \\
m \neq n}}^{+\infty} e^{-i k n d} K_{\alpha}(n-m) u_{m} .
\end{gathered}
$$

Using conditions (15), we introduce the notations

$$
\begin{aligned}
\widehat{K}_{\alpha}(k d) & =\sum_{\substack{n=-\infty \\
n \neq 0}}^{+\infty} e^{-i k n d} K_{\alpha}(n), \\
\widehat{u}(k, t) & =\sum_{n=-\infty}^{+\infty} e^{-i k n d} u_{n}(t) .
\end{aligned}
$$

Using $K_{\alpha}(-n)=K_{\alpha}(n)$, the function (20) can be represented by

$$
\widehat{K}_{\alpha}(k d)=\sum_{n=1}^{+\infty} K_{\alpha}(n)\left(e^{-i k n d}+e^{i k n d}\right)=2 \sum_{n=1}^{+\infty} K_{\alpha}(n) \cos (k d) .
$$

From (22), we can see that $\widehat{K}_{\alpha}(k d)$ is a periodic function

$$
\widehat{K}_{\alpha}(k d+2 \pi m)=\widehat{K}_{\alpha}(k d),
$$

where $m$ is an integer. Using (21) and (20), the first term on the right-hand side of (19) gives

$$
\begin{aligned}
& \sum_{n=-\infty}^{+\infty} \sum_{\substack{m=-\infty \\
m \neq n}}^{+\infty} e^{-i k n d} K_{\alpha}(n-m) u_{n} \\
& =\sum_{n=-\infty}^{+\infty} e^{-i k n d} u_{n} \sum_{\substack{m^{\prime}=-\infty \\
m^{\prime} \neq 0}}^{+\infty} K_{\alpha}\left(m^{\prime}\right) \\
& =\widehat{u}(k, t) \widehat{K}_{\alpha}(0) .
\end{aligned}
$$

Here we use (15) and $K_{\alpha}\left(m^{\prime}+n-n\right)=K_{\alpha}\left(m^{\prime}\right)$ and

$$
\widehat{K}_{\alpha}(0)=\sum_{\substack{n=-\infty \\ n \neq 0}}^{+\infty} K_{\alpha}(n)=2 \sum_{n=1}^{\infty} K_{\alpha}(n)
$$


Using $K_{\alpha}\left(m, n^{\prime}+m\right)=K_{\alpha}\left(n^{\prime}\right)$, the second term on the righthand side of (19) has the form

$$
\begin{aligned}
& \sum_{n=-\infty}^{+\infty} \sum_{\substack{m=-\infty \\
m \neq n}}^{+\infty} e^{-i k n d} K_{\alpha}(n-m) u_{m} \\
& =\sum_{m=-\infty}^{+\infty} u_{m} \sum_{\substack{n=-\infty \\
n \neq m}}^{+\infty} e^{-i k n d} K_{\alpha}(n-m) \\
& =\sum_{m=-\infty}^{+\infty} u_{m} e^{-i k m d} \sum_{\substack{n^{\prime}=-\infty \\
n^{\prime} \neq n}}^{+\infty} e^{-i k n^{\prime} d} K_{\alpha}\left(n^{\prime}\right) \\
& =\widehat{u}(k, t) \widehat{K}_{\alpha}(k d) .
\end{aligned}
$$

Equations (24) and (26) give the expression

$$
\left(\widehat{K}_{\alpha}(0)-\widehat{K}_{\alpha}(k d)\right) \widehat{u}(k, t),
$$

where $\widehat{K}_{\alpha}(k d)$ is defined by (20).

Let us give the statement that describes the Fourier transform of the lattice equations.

Proposition 2. The Fourier series transform $\mathscr{F}_{\Delta}$ maps the lattice equations of motion

$$
\begin{aligned}
& M \frac{\partial^{2} u_{n}(t)}{\partial t^{2}} \\
& \quad=g \sum_{\substack{m=-\infty \\
m \neq n}}^{+\infty} K_{\alpha}(n-m)\left(u_{n}(t)-u_{m}(t)\right)+F(n),
\end{aligned}
$$

where $K_{\alpha}(n-m)$ satisfies conditions (15), into the continuum equation

$$
M \frac{\partial^{2} \widehat{u}(k, t)}{\partial t^{2}}=g\left(\widehat{K}_{\alpha}(0)-\widehat{K}_{\alpha}(k d)\right) \widehat{u}(k, t)+\mathscr{F}_{\Delta}\{F(n)\},
$$

where $\widehat{u}(k, t)=\mathscr{F}_{\Delta}\left\{u_{n}(t)\right\}, \widehat{K}_{\alpha}(k d)=\mathscr{F}_{\Delta}\left\{K_{\alpha}(n)\right\}$, and $\mathscr{F}_{\Delta}$ is an operator notation for the Fourier series transform.

Proof. To derive the equation for the field $\widehat{u}(k, t)$, we multiply (28) by $\exp (-i k n d)$ and sum over $n$ from $-\infty$ to $+\infty$. Then

$$
\begin{aligned}
& \sum_{n=-\infty}^{+\infty} e^{-i k n d} \frac{\partial^{2}}{\partial t^{2}} u_{n}(t) \\
& =g \sum_{n=-\infty}^{+\infty} \sum_{\substack{m=-\infty \\
m \neq n}}^{+\infty} e^{-i k n d} K_{\alpha}(n-m)\left(u_{n}-u_{m}\right) \\
& +\sum_{n=-\infty}^{+\infty} e^{-i k n d} F(n) .
\end{aligned}
$$

Using (21), the left-hand side of (30) has the form

$$
\sum_{n=-\infty}^{+\infty} e^{-i k n d} \frac{\partial^{2} u_{n}(t)}{\partial t^{2}}=\frac{\partial^{2}}{\partial t^{2}} \sum_{n=-\infty}^{+\infty} e^{-i k n d} u_{n}(t)=\frac{\partial^{2} \widehat{u}(k, t)}{\partial t^{2}}
$$

The second term of the right-hand side of (30) is

$$
\sum_{n=-\infty}^{+\infty} e^{-i k n d} F(n)=\mathscr{F}_{\Delta}\{F(n)\}
$$

The Fourier series transform $\mathscr{F}_{\Delta}$ maps the interaction term (16) into expression (17). As a result, we obtain (30) in the form (29), where $\mathscr{F}_{\Delta}\{F(n)\}$ is an operator notation for the Fourier series transform of $F(n)$.

\section{Fractional Weak Spatial Dispersion}

4.1. Weak Spatial Dispersion. Spatial dispersion is the dependence of $\widehat{K}_{\alpha}(|\mathbf{k}|)$ on the wave vector $\mathbf{k}$ that leads to nonlocal properties of the continuum. The spatial dispersion gives nonlocal connection between the stress tensor $\sigma_{k l}$ and the strain tensor $\varepsilon_{k l}$. The tensor $\sigma_{k l}$ at any point $\mathbf{r}$ of the continuum is not uniquely defined by the values of $\varepsilon_{k l}$ at this point. It also depends on the values of $\varepsilon_{k l}$ at neighboring points $\mathbf{r}^{\prime}$, located near the point $\mathbf{r}$.

A nonlocal constitutive relation between the stress $\sigma_{k l}$ and the strain $\varepsilon_{k l}$ can be understood on the basis of analysis of a lattice model. The particles of the lattice oscillate about their equilibrium positions and interact with each other. The equations of oscillations of the lattice particles with the local (nearest-neighbor) interaction give the partial differential equation of integer orders in the continuum limit $[23,24]$. Note that the lattice with nonlocal (long-range) interactions in the continuous limit can give fractional partial differential equations for nonlocal continuum $[23,24]$.

Qualitatively describing the process we can say that the fields of the elastic wave move particles from their equilibrium positions at a given point $\mathbf{r}$, which causes an additional shift of the particles in neighboring and more distant points $\mathbf{r}^{\prime}$ in some neighborhood. Therefore, the properties of the medium, and hence the stress tensor field $\sigma_{k l}$, depend on the values of strain tensor field $\varepsilon_{k l}$ not only in a selected point but also in its neighborhood.

The size of the area in which the kernel $\widehat{K}_{\alpha}(|\mathbf{k}|)$ is significant is determined by the characteristic length of interaction $R_{0}$. The size $R_{0}$ of the area is usually of the order of the lattice constant. Wavelength $\lambda$ of elastic wave is several orders larger than the size of this region, so the values of the field of elasticity wave do not change for a region of size $R_{0}$. In other words, the wavelength $\lambda$ usually holds $k R_{0} \sim R_{0} / \lambda \ll 1$. In such lattice, the spatial dispersion is weak. To describe the lattice dynamics, it is enough to know the dependence of the function $\widehat{K}_{\alpha}(|\mathbf{k}|)$ only for small values $k=|\mathbf{k}|$ and we can replace this function by the Taylors polynomial series. For an isotropic linear medium, we use

$$
\widehat{K}_{\alpha}(k)=\widehat{K}_{\alpha}(0)+a_{1} k+a_{2} k^{2}+\cdots .
$$


Here we neglect a frequency dispersion, and so $\widehat{K}_{\alpha}(0), a_{1}, a_{2}$ do not depend on the frequency $\omega$.

4.2. Fractional Taylor Series Approach. The weak spatial dispersion in the media with power-law type of nonlocality cannot be described by the usual Taylor approximation. The fractional Taylor series is very useful for approximating noninteger power-law functions [28]. For example, the usual Taylor series for the nonlinear power-law function

$$
\widehat{K}_{\alpha}(k)=a_{0}+a_{\alpha} k^{\alpha}
$$

has infinitely many terms for noninteger $\alpha$.

If we use the fractional Taylor's formula (see Appendix A) we get a finite number of terms. For example, Taylor's series in the Odibat-Shawagfeh form that contains the Caputo fractional derivative ${ }_{a}^{C} D_{k}^{\alpha}$ has two terms for (34). Using

$$
{ }_{0}^{C} D_{k}^{\alpha} k^{\beta}=\frac{\Gamma(\beta+1)}{\Gamma(\beta-\alpha+1)} k^{\beta-\alpha}, \quad(k>0, \alpha>0, \beta>0)
$$

for the case $\beta=\alpha$, we get

$$
{ }_{0}^{C} D_{k}^{\alpha} k^{\alpha}=\Gamma(\alpha+1), \quad\left({ }_{0}^{C} D_{k}^{\alpha}\right)^{n} k^{\alpha}=0 .
$$

As a result, we have

$$
\begin{gathered}
\left({ }_{0}^{C} D_{k}^{\alpha} \widehat{K}_{\alpha}\right)(0)=\Gamma(\alpha+1), \\
\left(\left({ }_{0}^{C} D_{k}^{\alpha}\right)^{n} \widehat{K}_{\alpha}\right)(0)=0, \quad(n \geq 2)
\end{gathered}
$$

and the fractional Taylor's series approximation of function (34) is exact.

4.3. Weak Spatial Dispersion of Power-Law Types. We consider properties of the lattice with weak spatial dispersion that is described by the function $\widehat{K}_{\alpha}(|\mathbf{k}|)$ of a noninteger powerlaw type. In the continuous limit this model gives a model of continuum with power-law nonlocality.

The Fourier series transform $\mathscr{F}_{\Delta}$ of the interaction term (16) is defined by (17), where

$$
\widehat{K}_{\alpha}(|\mathbf{k}|)=\sum_{\substack{n=-\infty \\ n \neq 0}}^{+\infty} e^{-i k n} K_{\alpha}(n)=2 \sum_{n=1}^{\infty} K_{\alpha}(n) \cos (n|\mathbf{k}|),
$$

and $\widehat{u}(k, t)=\mathscr{F}_{\Delta}\left\{u_{n}(t)\right\}$. If the function $\widehat{K}_{\alpha}(|\mathbf{k}|)$ is given, then $K_{\alpha}(n)$ can be defined by

$$
K_{\alpha}(n)=\frac{1}{\pi} \int_{0}^{\pi} \widehat{K}_{\alpha}(|\mathbf{k}|) \cos (n|\mathbf{k}|) d|\mathbf{k}| .
$$

The weak spatial dispersion will be called $\alpha_{1}$-type, if the function (38) satisfies condition

$$
\lim _{|\mathbf{k}| \rightarrow 0} \frac{\widehat{K}_{\alpha}(|\mathbf{k}|)-\widehat{K}_{\alpha}(0)}{|\mathbf{k}|^{\alpha_{1}}}=a_{\alpha_{1}}
$$

where $\alpha_{1}>0$ and $0<\left|a_{\alpha_{1}}\right|<\infty$. The weak spatial dispersion (and the interparticle interaction in the lattice) will be called $\alpha=\left(\alpha_{1}, \alpha_{2}\right)$-type, if the function $\widehat{K}_{\alpha}(|\mathbf{k}|)$ satisfies conditions $(40)$ and

$$
\lim _{|\mathbf{k}| \rightarrow 0} \frac{\widehat{K}_{\alpha}(|\mathbf{k}|)-\widehat{K}_{\alpha}(0)-a_{\alpha_{1}}|\mathbf{k}|^{\alpha_{1}}}{|\mathbf{k}|^{\alpha_{2}}}=a_{\alpha_{2}},
$$

where $\alpha_{2}>\alpha_{1}>0$ and $0<\left|a_{\alpha_{2}}\right|<\infty$.

Similarly we define the weak spatial dispersion and the interaction in the lattice of the $\alpha=\left(\alpha_{1}, \ldots, \alpha_{N}\right)$-type. For the weak spatial dispersion of the $\alpha=\left(\alpha_{1}, \ldots, \alpha_{N}\right)$-type, the function $\widehat{K}_{\alpha}(|\mathbf{k}|)$ can be represented in the form

$$
\widehat{K}_{\alpha}(|\mathbf{k}|)=\widehat{K}_{\alpha}(0)+\sum_{j=1}^{N} a_{\alpha_{j}}|\mathbf{k}|^{\alpha_{j}}+R_{\alpha}^{(N)}(|\mathbf{k}|),
$$

where $0<\alpha_{1}<\alpha_{2}<\cdots<\alpha_{N}$ and

$$
\lim _{|\mathbf{k}| \rightarrow 0} \frac{R_{\alpha}^{(N)}(|\mathbf{k}|)}{|\mathbf{k}|^{\alpha_{N}}}=0 .
$$

As a result, we can use the following approximation for weak spatial dispersion:

$$
\widehat{K}_{\alpha}(|\mathbf{k}|) \approx \widehat{K}_{\alpha}(0)+\sum_{j=1}^{N} a_{\alpha_{j}}|\mathbf{k}|^{\alpha_{j}} .
$$

If $\alpha_{j}=j$ for all $j \in \mathbb{N}$, we can use the usual Taylor's formula. In this case, we have the usual case of the weak spatial dispersion. In general, we should use a fractional generalization of Taylor's series (see Appendix A). If the orders of the fractional Taylor series approximation will be correlated with the type of weak spatial dispersion, then the fractional Taylor series approximation of $\widehat{K}_{\alpha}(|\mathbf{k}|)$ will be exact. In the general case $0<\alpha_{j+1}-\alpha_{j}<1$, we can use the fractional Taylor's formula in the Dzherbashyan-Nersesian form (see Appendix A). For the special cases $\alpha_{j}=j \alpha_{1}$, where $\alpha_{1}<1$ and/or $\alpha_{j}=\alpha+j$, we could use the other kind of the fractional Taylor's formulas.

\section{Fractional Gradient Elasticity Equation for Continuum}

In the continuous limit the equation for lattice with the interaction of the $\alpha$-type gives the equation for continuum of the fractional gradient model.

Proposition 3. In the continuous limit the lattice equation of motion

$$
M \frac{\partial^{2} u_{n}(t)}{\partial t^{2}}=g \sum_{\substack{m=-\infty \\ m \neq n}}^{+\infty} K_{\alpha}(n-m)\left(u_{n}(t)-u_{m}(t)\right)+F(n)
$$

with the weak spatial dispersion of the $\alpha$-type gives the fractional continuum equation of the form

$$
\frac{\partial^{2} u(x, t)}{\partial t^{2}}=-\sum_{j=1}^{N} G_{\alpha_{j}}\left((-\Delta)^{\alpha_{j} / 2} u\right)(x, t)+\frac{1}{\rho} f(x),
$$


where $(-\Delta)^{\alpha_{j} / 2}$ is the fractional Laplacian of order $\alpha_{j}$ in Riesz's form (see Appendix $B$ ), the variables $x$ and $d=d$ are dimensionless, $f(x)=F(x) /(A d), \rho=M /(A d)$, and

$$
G_{\alpha_{j}}=\frac{g a_{\alpha_{j}} d^{\alpha_{j}}}{M}, \quad(j=1, \ldots, N)
$$

are finite parameters.

Proof. The Fourier series transform $\mathscr{F}_{\Delta}$ of (45) gives (29). After division by the cross-sectional area of the medium $A$ and the interparticle distance $d$, the limit $d \rightarrow 0$ for (29) gives

$$
\frac{\partial^{2}}{\partial t^{2}} \widehat{u}(k, t)=\sum_{j=1}^{N} \frac{g d^{\alpha_{j}}}{M} \widehat{\mathscr{K}}_{\alpha_{j}, \Delta}(k) \widehat{u}(k, t)+\frac{1}{\rho} \mathscr{F}_{\Delta}\{f(n)\},
$$

where $\rho=M /(A d)$ is the mass density, $d$ is the interparticle distance, $f(n)=F(n) /(A d)$, and

$$
\widehat{\mathscr{K}}_{\alpha_{j}, \Delta}(k)=-a_{\alpha_{j}}|k|^{\alpha_{j}}-R_{\alpha}^{(N)}(k d) d^{-\alpha_{j}} .
$$

Here we use (44), and $G_{\alpha_{j}}(j=1, \ldots, N)$ are finite parameters that are defined by (47). Note that $R_{\alpha}^{(N)}$ satisfies the condition

$$
\lim _{d \rightarrow 0} \frac{R_{\alpha}^{(N)}(k d)}{d^{\alpha_{N}}}=0 .
$$

The expression for $\widehat{\mathscr{T}}_{\alpha_{j}, \Delta}(k)$ can be considered as a Fourier transform of the interaction term (see Proposition 1). Note that $g a_{\alpha_{j}} \rightarrow \infty$ for the limit $d \rightarrow 0$, if $G_{\alpha_{j}}$ are finite parameters.

$$
\begin{aligned}
& \text { In the limit } d \rightarrow 0 \text {, (48) gives } \\
& \frac{\partial^{2} \widetilde{u}(k, t)}{\partial t^{2}}=\sum_{j=1}^{N} G_{\alpha_{j}} \widehat{\mathscr{T}}_{\alpha_{j}}(k) \widetilde{u}(k, t)+\frac{1}{\rho} \mathscr{F}\{f(x)\},
\end{aligned}
$$

where

$$
\widehat{\mathscr{K}}_{\alpha_{j}}(k)=\mathscr{L} \widehat{\mathscr{K}}_{\alpha_{j}, \Delta}(k)=-a_{\alpha_{j}}|k|^{\alpha_{j}}, \quad \tilde{u}(k, t)=\mathscr{L} \widehat{u}(k, t) .
$$

The inverse Fourier transform of (51) has the form

$$
\frac{\partial^{2} u(x, t)}{\partial t^{2}}=\sum_{j=1}^{N} G_{\alpha_{j}} \mathscr{T}_{\alpha_{j}}(x) u(x, t)+\frac{1}{\rho} f(x),
$$

where

$$
\mathscr{T}_{\alpha_{j}}(x)=\mathscr{F}^{-1}\left\{\widehat{\mathscr{K}}_{\alpha_{j}}(k)\right\}=-a_{\alpha_{j}}(-\Delta)^{\alpha_{j} / 2} .
$$

Here, we use the connection between the Riesz fractional Laplacian $(-\Delta)^{\alpha / 2}$ and its Fourier transform (see Appendix B and $[1-3])$ :

$$
\mathscr{F}\left[(-\Delta)^{\alpha / 2} u(\mathbf{r})\right](\mathbf{k})=|\mathbf{k}|^{\alpha} \widehat{u}(\mathbf{k})
$$

in the form

$$
|k|^{\alpha_{j}} \longleftrightarrow(-\Delta)^{\alpha_{j} / 2}
$$

Substitution of (54) into (53) gives the continuum equation (46).
Equations (46) and (47) give the close relation between the discrete microstructure of lattice with weak spatial dispersion of power-law type and the fractional gradient models of weak nonlocal continuum.

Let us consider the special case $\alpha_{j}=j$ for integer $j \in \mathbb{N}$. If the function $\widehat{K}_{\alpha}(k)$ has the form

$$
\widehat{K}_{\alpha}(k) \approx \widehat{K}_{\alpha}(0)+a_{2} k^{2},
$$

then we get the well-known equation

$$
\frac{\partial^{2} u(x, t)}{\partial t^{2}}=G_{2} \Delta u(x, t)+\frac{1}{\rho} f(x) .
$$

Here

$$
G_{2}=\frac{g a_{2} d^{2}}{M A}=\frac{E}{\rho},
$$

where $E=K d / A$ is Young's modulus, $K=g a_{2}$ is the spring stiffness, and $\rho=M /(A d)$ is the mass density.

If we can use the spatial dispersion law in the form

$$
\widehat{K}_{\alpha}(k) \approx \widehat{K}_{\alpha}(0)+a_{2} k^{2}+a_{4} k^{4},
$$

then we have the equation of the gradient elasticity as

$$
\frac{\partial^{2} u(x, t)}{\partial t^{2}}=G_{2} \Delta u(x, t)-G_{4} \Delta^{2} u(x, t)+\frac{1}{\rho} f(x),
$$

where $\alpha_{j}=j$,

$$
G_{4}=\frac{g a_{4} d^{4}}{M A}=\frac{a_{4} E d^{2}}{a_{2} \rho}, \quad a_{j}=\left(\frac{\partial^{j} \widehat{K}_{\alpha}(k)}{\partial k^{j}}\right)_{k=0} .
$$

The scale parameter $l^{2}$ of the gradient elasticity is connected with the coupling constants of the lattice by the equation

$$
l^{2}=\frac{\left|a_{4}\right| d^{2}}{\left|a_{2}\right|} \text {. }
$$

The second-gradient term is preceded by the sign that is defined by $\operatorname{sgn}\left(a_{4} / a_{2}\right)$.

Similarly, we can consider more general model of lattice with fractional weak spatial dispersion of $\alpha=\left(\alpha_{1}, \ldots, \alpha_{N}\right)$ type

$$
\widehat{K}_{\alpha}(\mathbf{k})=\widehat{K}_{\alpha}(0)+\sum_{j=1}^{N} a_{\alpha_{j}}|\mathbf{k}|^{\alpha_{j}} .
$$

Then the continuum equation for fractional gradient model has the form

$$
\frac{\partial^{2} u(\mathbf{r}, t)}{\partial t^{2}}=-\sum_{j=1}^{N} c_{j}\left((-\Delta)^{\alpha_{j} / 2} u\right)(\mathbf{r}, t)+\frac{1}{\rho} f(\mathbf{r}),
$$

where we use new notation for the constants, $c_{j}=G_{\alpha_{j}}$. Note that $\mathbf{r}$ and $r=|\mathbf{r}|$ are dimensionless. 


\section{Solution of Fractional Gradient Elasticity Equation}

6.1. Plane Wave Solution. Let us consider the plane waves $u(\mathbf{r}, t)=e^{-i \omega t} u(\mathbf{r})$. Then (65) gives

$$
\sum_{j=1}^{N} c_{j}\left((-\Delta)^{\alpha_{j} / 2} u\right)(\mathbf{r})-\omega^{2} u(\mathbf{r})=\frac{1}{\rho} f(\mathbf{r})
$$

We apply the Fourier method to solve fractional equation (66), which is based on the relation

$$
\mathscr{F}\left[(-\Delta)^{\alpha / 2} u(\mathbf{r})\right](\mathbf{k})=|\mathbf{k}|^{\alpha} \widehat{u}(\mathbf{k}) .
$$

Applying the Fourier transform $\mathscr{F}$ to both sides of (66) and using (67), we have

$$
(\mathscr{F} u)(\mathbf{k})=\frac{1}{\rho}\left(\sum_{j=1}^{N} c_{j}|\mathbf{k}|^{\alpha_{j}}-\omega^{2}\right)^{-1}(\mathscr{F} f)(\mathbf{k}) .
$$

The fractional analog of the Green function (see Section 5.5.1. in [3]) is given by

$$
\begin{aligned}
G_{\alpha}^{n}(\mathbf{r}) & =\mathscr{F}^{-1}\left[\left(\sum_{j=1}^{N} c_{j}|\mathbf{k}|^{\alpha_{j}}-\omega^{2}\right)^{-1}\right](\mathbf{r}) \\
& =\int_{\mathbb{R}^{n}}\left(\sum_{j=1}^{N} c_{j}|\mathbf{k}|^{\alpha_{j}}-\omega^{2}\right)^{-1} e^{+i(\mathbf{k}, \mathbf{r})} d^{n} \mathbf{k},
\end{aligned}
$$

where $\alpha=\left(\alpha_{1}, \ldots, \alpha_{m}\right)$.

The following relation

$$
\begin{aligned}
\int_{\mathbb{R}^{n}} e^{i(\mathbf{k}, \mathbf{r})} f(|\mathbf{k}|) d^{n} \mathbf{k} \\
\quad=\frac{(2 \pi)^{n / 2}}{|\mathbf{r}|^{(n-2) / 2}} \int_{0}^{\infty} f(\lambda) \lambda^{n / 2} J_{n / 2-1}(\lambda|\mathbf{r}|) d \lambda
\end{aligned}
$$

holds (see Lemma 25.1 of $[1,2]$ ) for any suitable function $f$ such that the integral in the right-hand side of (70) is convergent. Here $J_{v}$ is the Bessel function of the first kind. As a result, the Fourier transform of a radial function is also a radial function.

Using relation (70), the Green function (69) can be represented (see Theorem 5.22 in [3]) in the form of the integral with respect to one parameter:

$$
\begin{aligned}
G_{\alpha}^{n}(\mathbf{r})= & \frac{|\mathbf{r}|^{(2-n) / 2}}{(2 \pi)^{n / 2}} \\
& \times \int_{0}^{\infty}\left(\sum_{j=1}^{N} c_{j} \lambda^{\alpha_{j}}-\omega^{2}\right)^{-1} \lambda^{n / 2} J_{(n-2) / 2}(\lambda|\mathbf{r}|) d \lambda
\end{aligned}
$$

where $n=1,2,3$ and $\alpha=\left(\alpha_{1}, \ldots, \alpha_{m}\right)$ and $J_{(n-2) / 2}$ is the Bessel function of the first kind.
For the 3-dimensional case, we use

$$
J_{1 / 2}(z)=\sqrt{\frac{2}{\pi z}} \sin (z)
$$

Then we have

$$
G_{\alpha}^{3}(\mathbf{r})=\frac{1}{2 \pi^{2}|\mathbf{r}|} \int_{0}^{\infty}\left(\sum_{j=1}^{N} c_{j} \lambda^{\alpha_{j}}-\omega^{2}\right)^{-1} \lambda \sin (\lambda|\mathbf{r}|) d \lambda .
$$

For the 1-dimensional case, we use

$$
J_{-1 / 2}(z)=\sqrt{\frac{2}{\pi z}} \cos (z)
$$

Then we have (see Theorem 5.24 in [3] pages $345-346$ ) the function

$$
G_{\alpha}^{1}(\mathbf{r})=\frac{1}{\pi} \int_{0}^{\infty}\left(\sum_{s=1}^{m} a_{\alpha_{j}} \lambda^{\alpha_{j}}-\omega^{2}\right)^{-1} \cos (\lambda|\mathbf{r}|) d \lambda .
$$

If $\alpha_{N}>1$ and $c_{N} \neq 0$, then (66) (see, e.g., Section 5.5.1. pages 341-344 in [3]) has a particular solution $u(|\mathbf{r}|)$. Such particular solution is represented in the form of the convolution of the functions $G_{\alpha}^{n}(|\mathbf{r}|)$ and $f(|\mathbf{r}|)$ as follows:

$$
u(\mathbf{r})=\frac{1}{\rho} \int_{\mathbb{R}^{n}} G_{\alpha}^{n}\left(\mathbf{r}-\mathbf{r}^{\prime}\right) f\left(\mathbf{r}^{\prime}\right) d^{n} \mathbf{r}^{\prime},
$$

where the Green function $G_{\alpha}^{n}(\mathbf{r})$ is given by (71).

In 3-dimensional case, the function $f(|\mathbf{r}|)$ does not depend on the angles. Therefore, we can use the spherical coordinates and then reduce the integration $d^{3} \mathbf{r}^{\prime}$ in (76) to $d r=d|\mathbf{r}|$ by integrating with respect to the angles

$$
u(r)=\frac{4 \pi}{\rho} \int_{\mathbb{R}} G_{\alpha}^{3}\left(\left|\mathbf{r}-\mathbf{r}^{\prime}\right|\right) f\left(r^{\prime}\right)\left(r^{\prime}\right)^{2} d r^{\prime}
$$

where $r=|\mathbf{r}|$ and $r^{\prime}=\left|\mathbf{r}^{\prime}\right|$.

6.2. Static Solution. Let us consider the statics $(\partial u(\mathbf{r}, t) / \partial t=$ 0 , i.e., $u(\mathbf{r}, t)=u(\mathbf{r}))$ in the suggested fractional gradient elasticity model. We can consider the fractional partial differential equation (66) with $\omega^{2}=0$ and $c_{1} \neq 0$, when $N \geq 1$, and also the case where $\alpha_{1}<3, \alpha_{N}>1, N \geq 1, c_{1} \neq 0, c_{N} \neq 0$, $\alpha_{N}>\cdots>\alpha_{1}>0$, which is given by

$$
\sum_{j=1}^{N} c_{j}\left((-\Delta)^{\alpha_{j} / 2} u\right)(\mathbf{r})=\frac{1}{\rho} f(\mathbf{r}) .
$$

Equation (78) has the following particular solution (see Theorem 5.23 in [3]) that is represented in the form of the convolution of the functions as

$$
u(\mathbf{r})=\frac{1}{\rho} \int_{\mathbb{R}^{n}} G_{\alpha}^{n}\left(\mathbf{r}-\mathbf{r}^{\prime}\right) f\left(\mathbf{r}^{\prime}\right) d^{n} \mathbf{r}^{\prime}
$$


with the Green function

$$
G_{\alpha}^{n}(\mathbf{r})=\frac{|\mathbf{r}|^{(2-n) / 2}}{(2 \pi)^{n / 2}} \int_{0}^{\infty}\left(\sum_{j=1}^{N} c_{j} \lambda^{\alpha_{j}}\right)^{-1} \lambda^{n / 2} J_{(n-2) / 2}(\lambda|\mathbf{r}|) d \lambda,
$$

where $n=1,2,3$ and $\alpha=\left(\alpha_{1}, \ldots, \alpha_{m}\right)$.

These particular solutions allow us to describe static fields in the elastic continuum with the weak spatial dispersion of $\alpha=\left(\alpha_{1}, \ldots, \alpha_{N}\right)$-type.

\section{Fractional Weak Spatial Dispersion of $(\alpha, \beta)$-Type}

7.1. Fractional Gradient Elasticity Equation for Dispersion of $(\alpha, \beta)$-Type. If we have the dispersion law in the form

$$
\widehat{K}_{\alpha}(|\mathbf{k}|) \approx a_{\alpha}|\mathbf{k}|^{\alpha}+a_{\beta}|\mathbf{k}|^{\beta}+\widehat{K}_{\alpha}(0),
$$

where $\alpha>1, \beta<3$, and $0<\beta<\alpha$, then we have the fractional gradient elasticity equation

$$
c_{\alpha}\left((-\Delta)^{\alpha / 2} u\right)(\mathbf{r})+c_{\beta}\left((-\Delta)^{\beta / 2} u\right)(\mathbf{r})=\frac{1}{\rho} f(\mathbf{r}),
$$

where

$$
c_{\alpha}=\frac{g a_{\alpha} d^{\alpha}}{M}, \quad c_{\beta}=\frac{g a_{\beta} d^{\beta}}{M} .
$$

If $\alpha=4$ and $\beta=2$, we have the well-known equation of the gradient elasticity [15]:

$$
c_{2} \Delta u(\mathbf{r})-c_{4} \Delta^{2} u(\mathbf{r})+\frac{1}{\rho} f(\mathbf{r})=0,
$$

where

$$
c_{2}=\frac{E}{\rho}=\frac{g a_{2} d^{2}}{M}, \quad c_{4}= \pm l^{2} \frac{E}{\rho}=\frac{g a_{4} d^{4}}{M} .
$$

The second-gradient term is preceded by the sign that is defined by $\operatorname{sgn}\left(g a_{4}\right)$, where $g a_{2}>0$.

Equation (82) is the fractional partial differential equation (78) with $n=3$, and such equation has the particular solution [3] of the firm

$$
u(\mathbf{r})=\frac{1}{\rho} \int_{\mathbb{R}^{3}} G_{\alpha, \beta}^{3}\left(\mathbf{r}-\mathbf{r}^{\prime}\right) f\left(\mathbf{r}^{\prime}\right) d^{3} \mathbf{r}^{\prime},
$$

where the Green type function is given by

$$
G_{\alpha, \beta}^{3}(\mathbf{r})=\frac{|\mathbf{r}|^{-1 / 2}}{(2 \pi)^{3 / 2}} \int_{0}^{\infty}\left(c_{\alpha} \lambda^{\alpha}+c_{\beta}|\lambda|^{\beta}\right)^{-1} \lambda^{3 / 2} J_{1 / 2}(\lambda|\mathbf{r}|) d \lambda .
$$

Here $J_{1 / 2}$ is the Bessel function of the first kind.
7.2. Point Load Problem for Fractional Gradient Elasticity. Let us consider point load problem for an infinite elastic continuum (see pages 25-26 in [29]) and determine a deformation of an infinite gradient continuum, when a force is applied to a small region in it. We consider this Thomson's problem for nonlocal elastic continuum with fractional weak spatial dispersion of the form (81). If we consider the deformation at distances $|\mathbf{r}|$, which are larger than the size of the region, then we can assume that the force is applied at a point. In this case, we have

$$
f(\mathbf{r})=f_{0} \delta(\mathbf{r})=f_{0} \delta(x) \delta(y) \delta(z) .
$$

Then the displacement field $u(\mathbf{r})$ of fractional gradient elasticity has a simple form of the particular solution (79) that is proportional to the Greens function

$$
u(\mathbf{r})=\frac{f_{0}}{\rho} G_{\alpha}^{n}(\mathbf{r}),
$$

where $G_{\alpha}^{n}(z)$ is given by (80). Therefore, the displacement field (86) for the force that is applied at a point (88) has the form

$$
u(\mathbf{r})=\frac{1}{2 \pi^{2}} \frac{f_{0}}{\rho|\mathbf{r}|} \int_{0}^{\infty} \frac{\lambda \sin (\lambda|\mathbf{r}|)}{c_{\alpha} \lambda^{\alpha}+c_{\beta} \lambda^{\beta}} d \lambda
$$

From a mathematical point of view, there are two special cases: (1) fractional weak spatial dispersion of $(\alpha, \beta)$-type with $\alpha=2$ and $0<\beta<2$; (2) fractional weak spatial dispersion of $(\alpha, \beta)$-type with $\alpha \neq 2, \alpha>\beta$, and $0<\beta<3$.

From the point of view of the nonlocal elasticity theory, it is useful to distinguish the two following particular cases:

(i) subgradient elasticity $(\alpha=2$ and $0<\beta<2)$,

(ii) supergradient elasticity $(\alpha>2$ and $\beta=2)$.

Note that for the first case the order of the fractional Laplacian is less than the order of the first term related to the usual Hooke's law. In the second case the order of the fractional Laplacian is greater than the order of the first term related to the Hooke law. The names of the suband supergradient elasticity caused by the analogy with the names of anomalous diffusion [6-8] such as subdiffusion and superdiffusion.

7.3. Subgradient Elasticity Model. The subgradient elasticity is characterized by the fractional weak spatial dispersion of $(\alpha, \beta)$-type with $\alpha=2$ and $0<\beta<2$. Fractional model of nonlocal continuum with this spatial dispersion is described by (82) with $\alpha=2$ and $0<\beta<2$, given by

$$
c_{2} \Delta u(\mathbf{r})-c_{\beta}\left((-\Delta)^{\beta / 2} u\right)(\mathbf{r})+\frac{1}{\rho} f(\mathbf{r})=0, \quad(0<\beta<2) .
$$

The order of the fractional Laplacian $(-\Delta)^{\beta / 2}$ is less than the order of the first term related to the usual Hooke's law. As a simple example, consider the square of the Laplacian; that is, $\beta=1$. 
The particular solution of (91) for the force that is applied at a point (88) is the displacement field

$$
u(\mathbf{r})=\frac{1}{2 \pi^{2}} \frac{f_{0}}{\rho|\mathbf{r}|} \int_{0}^{\infty} \frac{\lambda \sin (\lambda|\mathbf{r}|)}{c_{2} \lambda^{2}+c_{\beta} \lambda^{\beta}} d \lambda .
$$

Using equation (1) of Section 2.3 in the book in [30, 31], we obtain the following asymptotic behavior for $u(|\mathbf{r}|)$ with $0<\beta<2$, when $|\mathbf{r}| \rightarrow \infty$ :

$$
\begin{aligned}
u(|\mathbf{r}|) & =\frac{f_{0}}{2 \pi^{2} \rho|\mathbf{r}|} \int_{0}^{\infty} \frac{\lambda \sin (\lambda|\mathbf{r}|)}{c_{2} \lambda^{2}+c_{\beta} \lambda^{\beta}} d \lambda \\
& \approx \frac{C_{0}(\beta)}{|\mathbf{r}|^{3-\beta}}+\sum_{k=1}^{\infty} \frac{C_{k}(\beta)}{|\mathbf{r}|^{(2-\beta)(k+1)+1}},
\end{aligned}
$$

where

$$
\begin{gathered}
C_{0}(\beta)=\frac{f_{0}}{2 \pi^{2} \rho c_{\beta}} \Gamma(2-\beta) \sin \left(\frac{\pi}{2} \beta\right), \\
C_{k}(\beta)=-\frac{f_{0} c_{2}^{k}}{2 \pi^{2} \rho c_{\beta}^{k+1}} \int_{0}^{\infty} z^{(2-\beta)(k+1)-1} \sin (z) d z .
\end{gathered}
$$

As a result, the displacement field for the force that is applied at a point in the continuum with this type of fractional weak spatial dispersion is given by

$$
u(\mathbf{r}) \approx \frac{C_{0}(\beta)}{|\mathbf{r}|^{3-\beta}}, \quad(0<\beta<2)
$$

on the long distance $|\mathbf{r}| \gg 1$.

7.4. Supergradient Elasticity Model. The supergradient elasticity is characterized by the fractional weak spatial dispersion of $(\alpha, \beta)$-type with $\alpha>2$ and $\beta=2$. For the nonlocal continuum with the weak spatial dispersion of the $(\alpha, \beta)$-type, where $\alpha>\beta>0,0<\beta<3$ and $\alpha \neq 2$, the displacement field for the fractional gradient model which is described by (82) includes two parameters $(\alpha, \beta)$. As an example of the nonlocal continuum with this type of spatial dispersion, we highlight the case of supergradient elasticity, where $\beta=2$ and $\alpha>2$. In this case (82) has the form

$$
c_{2} \Delta u(\mathbf{r})-c_{\alpha}\left((-\Delta)^{\alpha / 2} u\right)(\mathbf{r})+\frac{1}{\rho} f(\mathbf{r})=0, \quad(\alpha>2) .
$$

The order of the fractional Laplacian $(-\Delta)^{\alpha / 2}$ is greater than the order of the first term related to the Hooke law. If $\alpha=4$, (96) becomes (84). Therefore, the case $3<\alpha<5$ can be considered as close as possible $(\alpha \approx 4)$ to the usual gradient elasticity (84).

For the displacement field that is described by (82), where $\alpha>\beta>0,0<\beta<2$ and $\alpha \neq 2$ and the force $f(\mathbf{r})$ is applied at a point (88), we have the following asymptotic behavior:

$$
u(|\mathbf{r}|) \approx \frac{f_{0} \Gamma(2-\beta) \sin (\pi \beta / 2)}{2 \pi^{2} \rho c_{\beta}} \cdot \frac{1}{|\mathbf{r}|^{3-\beta}}, \quad(|\mathbf{r}| \longrightarrow \infty) .
$$

We note that this asymptotic behavior $|\mathbf{r}| \rightarrow \infty$ does not depend on the parameter $\alpha$. The field on the long distances is determined only by term with $(-\Delta)^{\beta / 2}(\alpha>\beta)$ that can be interpreted as a fractional nonlocal "deformation" of Hooke's law.

We note the existence of a maximum for the function $u(|\mathbf{r}|) \cdot|\mathbf{r}|$ in the case $0<\beta<2<\alpha$.

The asymptotic behavior of the displacement field $u(|\mathbf{r}|)$ for $|\mathbf{r}| \rightarrow 0$ is given by

$$
\begin{aligned}
& u(|\mathbf{r}|) \approx \frac{f_{0} \Gamma((3-\alpha) / 2)}{2^{\alpha} \pi^{2} \sqrt{\pi} \rho c_{\alpha} \Gamma(\alpha / 2)} \cdot \frac{1}{|\mathbf{r}|^{3-\alpha}}, \quad(1<\alpha<2), \\
& u(|\mathbf{r}|) \approx \frac{f_{0} \Gamma((3-\alpha) / 2)}{2^{\alpha} \pi^{2} \sqrt{\pi} \rho c_{\alpha} \Gamma(\alpha / 2)} \cdot|\mathbf{r}|^{\alpha-3}, \quad(2<\alpha<3), \\
& u(|\mathbf{r}|) \approx \frac{f_{0}}{2 \pi \alpha \rho c_{\beta}^{1-3 / \alpha} c_{\alpha}^{3 / \alpha} \sin (3 \pi / \alpha)}, \quad(\alpha>3),
\end{aligned}
$$

where we use Euler's reflection formula for Gamma function. The asymptotic relation (98) is not directly related to the supergradient case. Note that the above asymptotic behavior does not depend on the parameter $\beta$, and relations (98)(99) do not depend on $c_{\beta}$. The displacement field $u(|\mathbf{r}|)$ on the short distances is determined only by term with $(-\Delta)^{\alpha / 2}$ $(\alpha>\beta)$ that can be considered as a fractional nonlocal "deformation" of the gradient term.

\section{Conclusion}

A lattice model with spatial dispersion of power-law type is suggested. Gradient elasticity is considered as a phenomenological theory representing continuum limit of lattice dynamics, where the length scales are much larger than interatomic distances. In the continuum limit we derive continuum equations with spatial derivatives of noninteger order $\alpha$. The correspondent continuum equations describe fractional generalization of gradient elasticity (the supergradient elasticity model) for $\alpha>2$ and a special form of fractional integral elasticity (the subgradient elasticity model) for $0<\alpha<2$. The suggested lattice model with spatial dispersion can be considered as a microscopic basis for the fractional nonlocal elastic continuum. We can note that a fractional nonlocal continuum model can be obtained from different microscopic or lattice models [32, 33]. The main advantage of the suggested approach is that we can use the Taylor series in the wave vector space instead of Taylor expansion in a coordinate space. It allows us to use these models as a microstructural basis of unified description of fractional (and integer) gradient models with positive and negative signs of the strain gradient terms. The suggested approach can be generalized for three-dimensional case of gradient elasticity. The proposed lattice model can also be easily generalized for the case of the high-order gradient elasticity and the correspondent fractional extension by using the next terms of fractional Taylor series. The suggested lattice models with long-range interactions can be important to describe the nonlocal elasticity of materials at microscale and 
nanoscales [34-36], where the interatomic and intermolecular interactions are prevalent in determining the properties of these materials.

\section{Appendices}

\section{A. Fractional Taylor Formula}

A.1. Riemann-Liouville and Caputo Derivatives. The leftsided Riemann-Liouville derivatives of order $\alpha>0$ are defined by

$$
\begin{aligned}
\left({ }^{\mathrm{RL}} D_{a+}^{\alpha} f\right)(x)= & \frac{1}{\Gamma(n-\alpha)}\left(\frac{d}{d x}\right)^{n} \\
& \times \int_{a}^{x} \frac{f\left(x^{\prime}\right) d x^{\prime}}{\left(x-x^{\prime}\right)^{\alpha-n+1}}, \quad(n=[\alpha]+1) .
\end{aligned}
$$

We can rewrite this relation in the form

$$
\left({ }^{\mathrm{RL}} D_{a+}^{\alpha} f\right)(x)=\left(\frac{d}{d x}\right)^{n}\left(I_{a+}^{n-\alpha} f\right)(x),
$$

where $I_{a+}^{\alpha}$ is a left-sided Riemann-Liouville integral of order $\alpha>0$ :

$$
\left(I_{a+}^{\alpha} f\right)(x)=\frac{1}{\Gamma(\alpha)} \int_{a}^{x} \frac{f\left(x^{\prime}\right) d x^{\prime}}{\left(x-x^{\prime}\right)^{1-\alpha}}, \quad(x>a) .
$$

The Caputo fractional derivative of order $\alpha$ is defined by

$$
\left({ }^{C} D_{a+}^{\alpha} f\right)(x)=\left(I_{a+}^{n-\alpha}\left(\frac{d}{d x}\right)^{n} f\right)(x),
$$

where $I_{a+}^{\alpha}$ is a left-sided Riemann-Liouville integral (A.3) of order $\alpha>0$. In (A.16) we use $0<\alpha<1$ and $n=1$. The main distinguishing feature of the Caputo fractional derivative is that, like the integer order derivative, the Caputo fractional derivative of a constant is zero.

Note also that the third term in (A.16) involves the fractional derivative of the fractional derivative, which is not the same as the $2 \alpha$ fractional derivative. In general,

$$
\left({ }^{C} D_{a+}^{\alpha}{ }^{C} D_{a+}^{\alpha} f\right)(x) \neq\left({ }^{C} D_{a+}^{2 \alpha} f\right)(x) .
$$

Then the coefficients of the fractional Taylor series can be found in the usual way, by repeated differentiation. This is to ensure that the fractional derivative of order $\alpha$ of the function $(x-a)^{\alpha}$ is a constant. The repeated fractional derivative of order $\alpha$ gives zero. Then the coefficients of the fractional Taylor series can be found in the usual way, by repeated differentiation.

A.2. Fractional Taylor's Series in the Riemann-Liouville Form. Let $f(x)$ be a real-value function such that the derivative $\left({ }^{\mathrm{RL}} D_{a+}^{\alpha+m} f\right)(x)$ is integrable. Then the following analog of Taylor formula holds (see Chapter 1. Section $2.6[1,2]$ ):

$$
f(x)=\sum_{j=0}^{m-1} \frac{\left({ }^{\mathrm{RL}} D_{a+}^{\alpha+j} f\right)(a+)}{\Gamma(\alpha+j+1)}(x-a)^{\alpha+j}+R_{m}(x),
$$

$(\alpha>0)$,

where $D_{a+}^{\alpha+j}$ are left-sided Riemann-Liouville derivatives and

$$
R_{m}(x)=\left(I_{a+}^{\alpha+m R L} D_{a+}^{\alpha+m} f\right)(x) .
$$

A.3. Riemann Formal Version of the Generalized Taylor's Series. The Riemann formal version of the generalized Taylor's series [37-39] is

$$
f(x)=\sum_{m=-\infty}^{+\infty} \frac{\left({ }^{\mathrm{RL}} D_{a}^{\alpha+m} f\right)\left(x_{0}\right)}{\Gamma(\alpha+m+1)}\left(x-x_{0}\right)^{\alpha+m},
$$

where ${ }^{\mathrm{RL}} D_{a}^{\alpha}$ for $\alpha>0$ is the Riemann-Liouville fractional derivative and ${ }^{\mathrm{RL}} D_{a}^{\alpha}=I_{a}^{-\alpha}$ for $\alpha<0$ is the RiemannLiouville fractional integral of order $|\alpha|$.

A.4. Fractional Taylor's Series in the Trujillo-Rivero-Bonilla Form. The Trujillo-Rivero-Bonilla form of generalized Taylor's formula [40] is

$$
f(x)=\sum_{j=0}^{m} \frac{c_{j}}{\Gamma((j+1) \alpha)}(x-a)^{(j+1) \alpha-1}+R_{m}(x, a),
$$

where $\alpha \in[0 ; 1]$ and

$$
\begin{gathered}
c_{j}=\Gamma(\alpha)\left[(x-a)^{1-\alpha}\left({ }^{\mathrm{RL}} D_{a}^{\alpha}\right)^{j} f(x)\right](a+), \\
R_{m}(x, a)=\frac{\left(\left({ }^{\mathrm{RL}} D_{a}^{\alpha}\right)^{m+1} f\right)(\xi)}{\Gamma((m+1) \alpha+1)}(x-a)^{(m+1) \alpha}, \quad \xi \in[a ; x] .
\end{gathered}
$$

A.5. Fractional Taylor's Series in the Dzherbashyan-Nersesian Form. Let $\alpha_{k}(k=0,1, \ldots, m)$ be increasing sequence of real numbers such that

$$
0<\alpha_{k}-\alpha_{k-1} \leq 1, \quad \alpha_{0}=0, \quad k=1,2, \ldots, m .
$$

We introduce the following notation [41, 42] (see also Section 2.8 in $[1,2])$ :

$$
D^{\left(\alpha_{k}\right)}=I_{0+}^{1-\left(\alpha_{k}-\alpha_{k-1}\right)} D_{0+}^{1+\alpha_{k-1}} .
$$

In general, $D^{\left(\alpha_{k}\right)} \neq{ }^{\mathrm{RL}} D_{0+}^{\alpha_{k}}$. Fractional derivative $D^{\left(\alpha_{k}\right)}$ differs from the Riemann-Liouville derivative ${ }^{\mathrm{RL}} D_{0+}^{\alpha_{k}}$ by finite sum of power functions since (see equation (2.68) in [3])

$$
I_{0+}^{\alpha} I_{0+}^{\beta} \neq I_{0+}^{\alpha+\beta} .
$$

The generalized Taylor's formula $[41,42]$ is

$$
f(x)=\sum_{k=0}^{m-1} a_{k} x^{\alpha_{k}}+R_{m}(x), \quad(x>0),
$$


where

$$
\begin{aligned}
a_{k} & =\frac{\left(D^{\left(\alpha_{k}\right)} f\right)(0)}{\Gamma\left(\alpha_{k}+1\right)} \\
R_{m}(x) & =\frac{1}{\Gamma\left(\alpha_{m}+1\right)} \int_{0}^{x}(x-z)^{\alpha_{m}-1}\left(D^{\left(\alpha_{k}\right)} f\right)(z) d z .
\end{aligned}
$$

A.6. Fractional Taylor's Series in the Odibat-Shawagfeh Form. The fractional Taylor series is a generalization of the Taylor series for fractional derivatives, where $\alpha$ is the fractional order of differentiation, $0<\alpha<1$. The fractional Taylor series with Caputo derivatives [43] has the form

$$
\begin{aligned}
f(x)= & f(a)+\frac{\left({ }^{C} D_{a+}^{\alpha} f\right)(a)}{\Gamma(\alpha+1)}(x-a)^{\alpha} \\
& +\frac{\left({ }^{C} D_{a+}^{\alpha}{ }^{C} D_{a+}^{\alpha} f\right)(a)}{\Gamma(2 \alpha+1)}(x-a)^{2 \alpha}+\cdots,
\end{aligned}
$$

where ${ }^{C} D_{a+}^{\alpha}$ is the Caputo fractional derivative of order $\alpha$.

\section{B. Riesz Fractional Derivatives and Integrals}

Fractional integration and fractional differentiation in the $n$ dimensional Euclidean space $\mathbb{R}^{n}$ can be defined as fractional powers of the Laplace operator. For $\alpha>0$ and "sufficiently good" functions $f(x), x \in \mathbb{R}^{n}$, the fractional Laplacian in Riesz's form (the Riesz fractional derivative) is defined in terms of the Fourier transform $\mathscr{F}$ by

$$
\left((-\Delta)^{\alpha / 2} f\right)(x)=\mathscr{F}^{-1}\left(|k|^{\alpha}(\mathscr{F} f)(k)\right) .
$$

The Riesz fractional integration is defined by

$$
\mathbf{I}_{x}^{\alpha} f(x)=\mathscr{F}^{-1}\left(|k|^{-\alpha}(\mathscr{F} f)(k)\right) .
$$

The Riesz fractional integration can be realized in the form of the Riesz potential defined as Fourier's convolution of the form

$$
\mathbf{I}_{x}^{\alpha} f(x)=\int_{\mathbb{R}^{n}} K_{\alpha}(x-z) f(z) d z, \quad(\alpha>0),
$$

where the function $K_{\alpha}(x)$ is the Riesz kernel. If $\alpha>0$ and $\alpha \neq n, n+2, n+4, \ldots$, the function $K_{\alpha}(x)$ is defined by

$$
K_{\alpha}(x)=\gamma_{n}^{-1}(\alpha)|x|^{\alpha-n} .
$$

If $\alpha \neq n, n+2, n+4, \ldots$, then

$$
K_{\alpha}(x)=-\gamma_{n}^{-1}(\alpha)|x|^{\alpha-n} \ln |x| .
$$

The constant $\gamma_{n}(\alpha)$ has the form

$$
\gamma_{n}(\alpha)=\left\{\begin{array}{l}
\frac{2^{\alpha} \pi^{n / 2} \Gamma(\alpha / 2)}{\Gamma((n-\alpha) / 2)} \\
\quad \alpha \neq n+2 k, \quad n \in \mathbb{N} \\
(-1)^{(n-\alpha) / 2} 2^{\alpha-1} \pi^{n / 2} \Gamma\left(\frac{\alpha}{2}\right) \Gamma\left(1+\frac{[\alpha-n]}{2}\right), \\
\alpha=n+2 k
\end{array}\right.
$$

Obviously, the Fourier transform of the Riesz fractional integration is given by

$$
\mathscr{F}\left(\mathbf{I}_{x}^{\alpha} f(x)\right)=|k|^{-\alpha}(\mathscr{F} f)(k) .
$$

This formula is true for functions $f(x)$ belonging to Lizorkin's space. The Lizorkin space of test functions on $\mathbb{R}^{n}$ is a linear space of all complex-valued infinitely differentiable functions $f(x)$ whose derivatives vanish at the origin:

$$
\Psi=\left\{f(x): f(x) \in S\left(\mathbb{R}^{n}\right),\left(D_{x}^{\mathbf{n}} f\right)(0)=0,|\mathbf{n}| \in \mathbb{N}\right\}
$$

where $S\left(\mathbb{R}^{n}\right)$ is the Schwartz test-function space. The Lizorkin space is invariant with respect to the Riesz fractional integration. Moreover, if $f(x)$ belongs to the Lizorkin space, then

$$
\mathbf{I}_{x}^{\alpha} \mathbf{I}_{x}^{\beta} f(x)=\mathbf{I}_{x}^{\alpha+\beta} f(x),
$$

where $\alpha>0$ and $\beta>0$.

For $\alpha>0$, the fractional Laplacian in Riesz's form can be defined in the form of the hypersingular integral by

$$
\left((-\Delta)^{\alpha / 2} f\right)(x)=\frac{1}{d_{n}(m, \alpha)} \int_{\mathbb{R}^{n}} \frac{1}{|z|^{\alpha+n}}\left(\Delta_{z}^{m} f\right)(z) d z,
$$

where $m>\alpha$ and $\left(\Delta_{z}^{m} f\right)(z)$ is a finite difference of order $m$ of a function $f(x)$ with a vector step $z \in \mathbb{R}^{n}$ and centered at the point $x \in \mathbb{R}^{n}$ :

$$
\left(\Delta_{z}^{m} f\right)(z)=\sum_{k=0}^{m}(-1)^{k} \frac{m !}{k !(m-k) !} f(x-k z) .
$$

The constant $d_{n}(m, \alpha)$ is defined by

$$
d_{n}(m, \alpha)=\frac{\pi^{1+n / 2} \mathrm{~A}_{m}(\alpha)}{2^{\alpha} \Gamma(1+\alpha / 2) \Gamma(n / 2+\alpha / 2) \sin (\pi \alpha / 2)},
$$

where

$$
A_{m}(\alpha)=\sum_{j=0}^{m}(-1)^{j-1} \frac{m !}{j !(m-j) !} j^{\alpha} .
$$

Note that the hypersingular integral $\left((-\Delta)^{\alpha / 2} f\right)(x)$ does not depend on the choice of $m>\alpha$.

If $f(x)$ belongs to the space of "sufficiently good" functions, then the Fourier transform $\mathscr{F}$ of the fractional Laplacian in Riesz's form is given by

$$
\left(\mathscr{F}(-\Delta)^{\alpha / 2} f\right)(k)=|k|^{\alpha}(\mathscr{F} f)(k) .
$$

This equation is valid for the Lizorkin space $[1,2]$ and the space $C^{\infty}\left(\mathbb{R}^{n}\right)$ of infinitely differentiable functions on $\mathbb{R}^{n}$ with compact support.

The fractional Laplacian in Riesz's form yields an operator inverse to the Riesz fractional integration for a special space of functions. The formula

$$
(-\Delta)^{\alpha / 2} \mathbf{I}_{x}^{\alpha} f(x)=f(x), \quad(\alpha>0)
$$

holds for "sufficiently good" functions $f(x)$. In particular, (B.15) for $f(x)$ belongs to the Lizorkin space. Moreover, this property is also valid for the Riesz fractional integration in the frame of $L_{p}$-spaces: $f(x) \in L_{p}(\mathbb{R})$ for $1 \leqslant p<n / a$ (see Theorem 26.3 in $[1,2])$. 


\section{Conflict of Interests}

The author declares that there is no conflict of interests regarding the publication of this paper.

\section{Acknowledgments}

The author expresses his gratitude to Professor Elias C. Aifantis for valuable discussions of fractional gradient elasticity and to Professor Juan J. Trujillo for valuable discussions of applications of fractional models in elasticity theory.

\section{References}

[1] S. G. Samko, A. A. Kilbas, and O. I. Marichev, Integrals and Derivatives of Fractional Order and Applications, Nauka i Tehnika, Minsk, Russia, 1987.

[2] S. G. Samko, A. A. Kilbas, and O. I. Marichev, Fractional Integrals and Derivatives Theory and Applications, Gordon and Breach, New York, NY, USA, 1993.

[3] A. A. Kilbas, H. M. Srivastava, and J. J. Trujillo, Theory and Applications of Fractional Differential Equations, Elsevier, Amsterdam, The Netherlands, 2006.

[4] A. Carpinteri and F. Mainardi, Eds., Fractals and Fractional Calculus in Continuum Mechanics, Springer, New York, NY, USA, 1997.

[5] R. Hilfer, Ed., Applications of Fractional Calculus in Physics, World Scientific, Singapore, 2000.

[6] R. Metzler and J. Klafter, “The random walk's guide to anomalous diffusion: a fractional dynamics approach," Physics Report, vol. 339, no. 1, pp. 1-77, 2000.

[7] G. M. Zaslavsky, "Chaos, fractional kinetics, and anomalous transport," Physics Report, vol. 371, no. 6, pp. 461-580, 2002.

[8] R. Metzler and J. Klafter, "The restaurant at the end of the random walk: recent developments in the description of anomalous transport by fractional dynamics," Journal of Physics A: Mathematical and General, vol. 37, no. 31, pp. R161-R208, 2004.

[9] J. Sabatier, O. P. Agrawal, and J. A. Tenreiro Machado, Eds., Advances in Fractional Calculus. Theoretical Developments and Applications in Physics and Engineering, Springer, Dordrecht, The Netherlands, 2007.

[10] A. C. J. Luo and V. S. Afraimovich, Eds., Long-Range Interaction, Stochasticity and Fractional Dynamics, Springer, Berlin, Germany, 2010.

[11] F. Mainardi, Fractional Calculus and Waves in Linear Viscoelasticity: An Introduction To Mathematical Models, World Scientific, Singapore, 2010.

[12] J. Klafter, S. C. Lim, and R. Metzler, Eds., Fractional Dynamics. Recent Advances, World Scientic, Singapore, 2011.

[13] V. E. Tarasov, Fractional Dynamics: Applications of Fractional Calculus To Dynamics of Particles, Fields and Media, Springer, New York, NY, USA, 2011.

[14] V. E. Tarasov, "Review of some promising fractional physical models," International Journal of Modern Physics B, vol. 27, no. 9, Article ID 133000, 32 pages, 2013.

[15] H. Askes and E. C. Aifantis, "Gradient elasticity in statics and dynamics: an overview of formulations, length scale identification procedures, finite element implementations and new results," International Journal of Solids and Structures, vol. 48, no. 13, pp. 1962-1990, 2011.
[16] K. A. Lazopoulos, "Non-local continuum mechanics and fractional calculus," Mechanics Research Communications, vol. 33, no. 6, pp. 753-757, 2006.

[17] A. Carpinteri, P. Cornetti, and A. Sapora, "Static-kinematic fractional operators for fractal and non-loeal solids," ZAMM Zeitschrift fur Angewandte Mathematik und Mechanik, vol. 89, no. 3, pp. 207-217, 2009.

[18] A. Carpinteri, P. Cornetti, and A. Sapora, "A fractional calculus approach to nonlocal elasticity," European Physical Journal: Special Topics, vol. 193, no. 1, pp. 193-204, 2011.

[19] A. Sapora, P. Cornetti, and A. Carpinteri, "Wave propagation in nonlocal elastic continua modelled by a fractional calculus approach," Communications in Nonlinear Science and Numerical Simulation, vol. 18, no. 1, pp. 63-74, 2013.

[20] G. Cottone, M. Di Paola, and M. Zingales, "Elastic waves propagation in 1D fractional non-local continuum," Physica E: Low-Dimensional Systems and Nanostructures, vol. 42, no. 2, pp. 95-103, 2009.

[21] G. Cottone, M. D. Paola, and M. Zingales, "Fractional mechanical model for the dynamics of non-local continuum," Lecture Notes in Electrical Engineering, vol. 11, pp. 389-423, 2009.

[22] P. Cornetti, A. Carpinteri, A. Sapora, M. Di Paola, and M. Zingales, "An explicit mechanical interpretation of Eringen non-local elasticity by means of fractional calculus," http://www.dipmat.univpm.it/.

[23] V. E. Tarasov, "Continuous limit of discrete systems with longrange interaction," Journal of Physics A: Mathematical and General, vol. 39, no. 48, article 5, pp. 14895-14910, 2006.

[24] V. E. Tarasov, "Map of discrete system into continuous," Journal of Mathematical Physics, vol. 47, no. 9, Article ID 092901, 2006.

[25] V. E. Tarasov and G. M. Zaslavsky, "Fractional dynamics of coupled oscillators with long-range interaction," Chaos, vol. 16, no. 2, 13 pages, 2006.

[26] N. Laskin and G. Zaslavsky, "Nonlinear fractional dynamics on a lattice with long range interactions," Physica A: Statistical Mechanics and its Applications, vol. 368, no. 1, pp. 38-54, 2006.

[27] V. E. Tarasov and G. M. Zaslavsky, "Fractional dynamics of systems with long-range interaction," Communications in Nonlinear Science and Numerical Simulation, vol. 11, no. 8, pp. 885-898, 2006.

[28] V. E. Tarasov and J. J. Trujillo, "Fractional power-law spatial dispersion in electrodynamics," Annals of Physics, vol. 334, pp. $1-23,2013$.

[29] L. D. Landau and E. M. Lifshitz, Theory of Elasticity, Oxford, UK, 1986.

[30] H. Bateman and A. Erdelyi, Tables of Integral Transforms Volume 1, McGraw-Hill, New York, NY, USA, 1954.

[31] H. Bateman and A. Erdelyi, Tables of Integral Transforms Volume 1, Nauka, Moscow, Russia, 1969, Russian.

[32] V. E. Tarasov, "Lattice model with power-law spatial dispersion for fractional elasticity," Central European Journal of Physics, vol. 11, no. 11, pp. 1580-1588, 2013.

[33] V. E. Tarasov, "Lattice model of fractional gradient and integral elasticity: long-range interaction of Grunwald-Letnikov-Riesz type," Mechanics of Materials, vol. 70, no. 1, pp. 106-114, 2014.

[34] L. Merchan, R. Szoszkiewicz, and E. Riedo, "NanoMechanics: elasticity in nano-objects," in Fundamentals of Friction and Wear NanoScience and Technology, E. Gnecco and E. Meyer, Eds., pp. 219-254, Springer, 2007.

[35] E. C. Aifantis, "Gradient effects at macro, micro, and nano scales," Journal of the Mechanical Behavior of Materials, vol. 5, no. 3, pp. 355-375, 1994. 
[36] E. C. Aifantis, "Gradient nanomechanics: applications to deformation, fracture, and diffusion in nanopolycrystals," Metallurgical and Materials Transactions A: Physical Metallurgy and Materials Science, vol. 42, no. 10, pp. 2985-2998, 2011.

[37] B. Riemann, Versuch Einer Allgemeinen Auffassung Der Integration Und Di Erentiation, Gesammelte MathematischeWerke und Wissenschaftlicher, Leipzig, Germany, 1876.

[38] B. Riemann, Versuch Einer Allgemeinen Auffassung Der Integration Und Di Erentiation, Dover, New York, NY, USA, 1953, German.

[39] G. H. Hardy, "Riemann's form of Taylor series," Journal of the London Mathematical Society, vol. 20, pp. 45-57, 1945.

[40] J. J. Trujillo, M. Rivero, and B. Bonilla, "On a Riemann-Liouville Generalized Taylor's Formula," Journal of Mathematical Analysis and Applications, vol. 231, no. 1, pp. 255-265, 1999.

[41] M. M. Dzherbashyan and A. B. Nersesian, The Criterion of the Expansion of the Functions To Dirichlet Series, Izvestiya Akademii Nauk Armyanskoi SSR. Seriya FizikoMatematicheskih Nauk, 1958, Russian.

[42] M. M. Dzherbashyan and A. B. Nersesian, "About application of some integro-differential operators," in Proceedings of the Russian Academy of Sciences, vol. 121, pp. 210-213, Doklady Akademii Nauk, Russian, 1958.

[43] Z. M. Odibat and N. T. Shawagfeh, "Generalized Taylor's formula," Applied Mathematics and Computation, vol. 186, no. 1, pp. 286-293, 2007. 

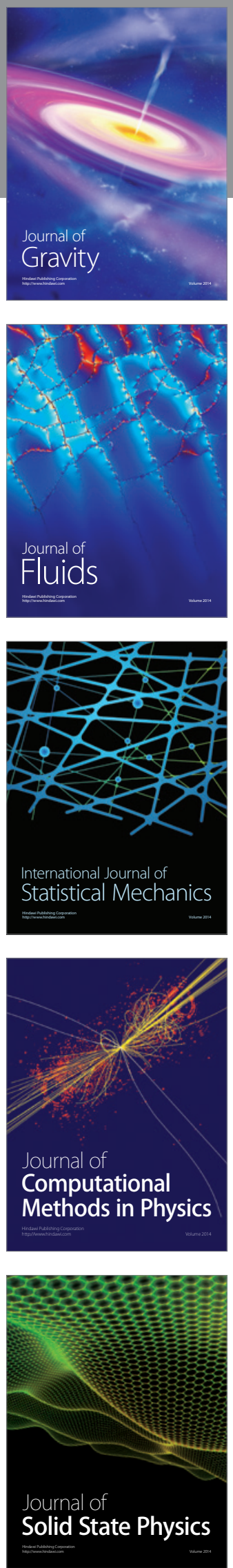

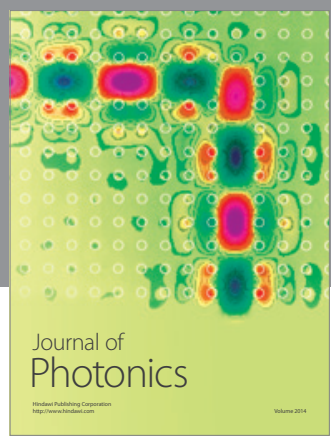

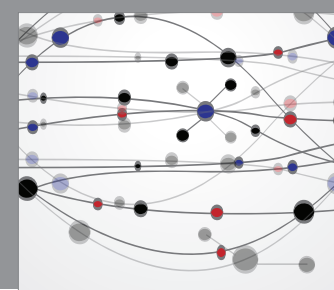

The Scientific World Journal

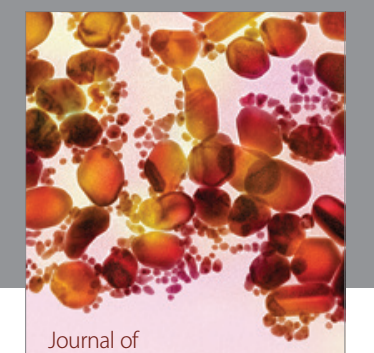

Soft Matter
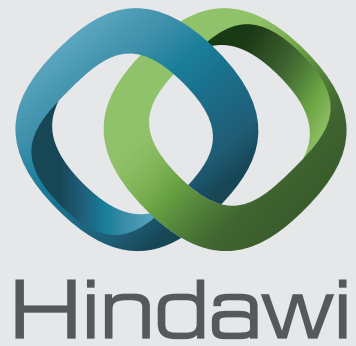

Submit your manuscripts at

http://www.hindawi.com
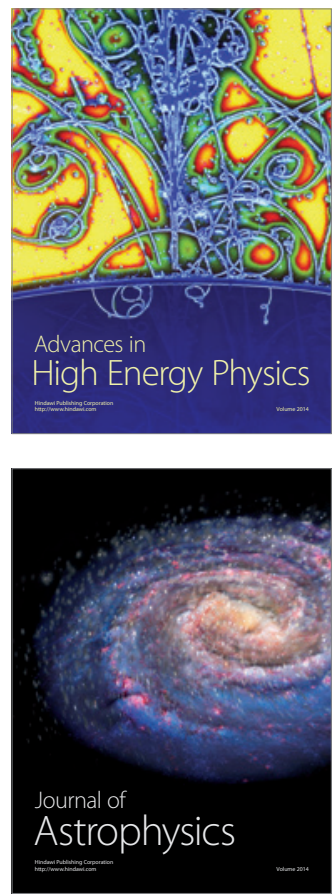
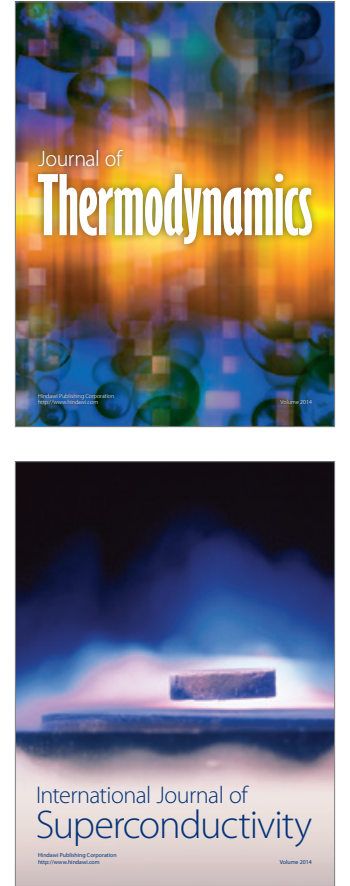
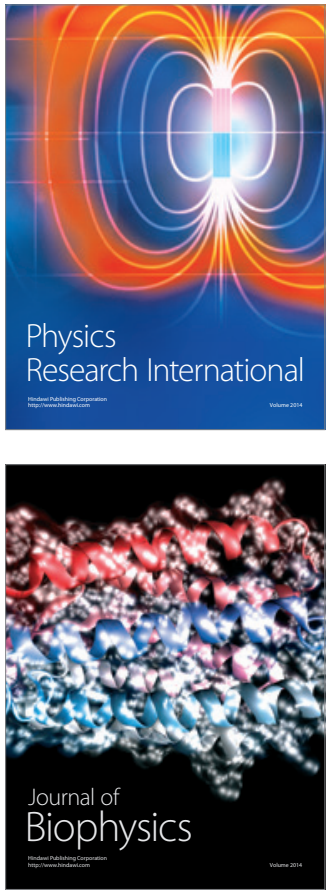
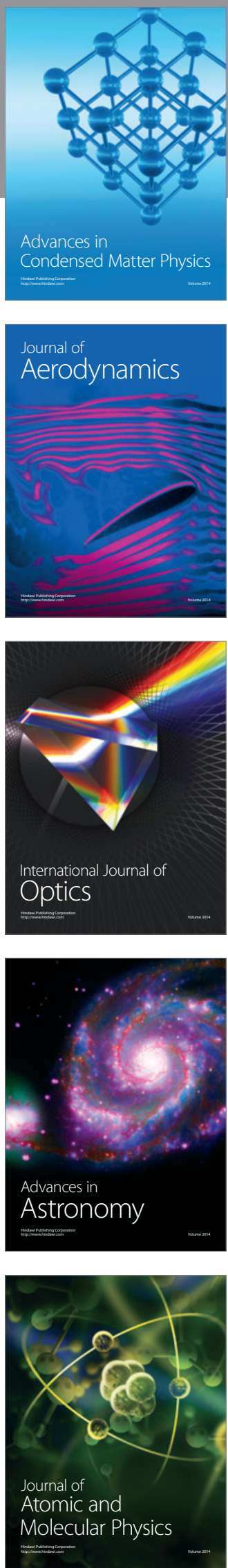